\title{
Letter migration errors reflect spatial pooling of orthographic information
}

\author{
Aaron Vandendaele ${ }^{1} \cdot$ Joshua Snell ${ }^{2,3} \cdot$ Jonathan Grainger ${ }^{2,3}$ \\ Published online: 6 May 2019 \\ (C) The Author(s) 2019
}

\begin{abstract}
Prior research has shown that readers may misread words by switching letters across words (e.g., the word sand in sand lane being recognized as land). These so-called letter migration errors have been observed using a divided attention paradigm whereby two words are briefly presented simultaneously, and one is postcued for identification. Letter migrations might therefore be due to a task-induced division of attention across the two words. Here, we show that a similar rate of migration errors is obtained in a flanker paradigm in which a central target word is flanked to the left and to the right by task-irrelevant flanking words. Three words were simultaneously presented for the same brief duration. Asked to type the target word postoffset, participants produced more migration errors when the migrating letter occupied the same position in the flanker and target words, with significantly fewer migrations occurring across adjacent positions, and the effect disappearing across nonadjacent positions. Our results provide further support for the hypothesis that orthographic information spanning multiple words is processed in parallel and spatially integrated (pooled) within a single channel. It is the spatial pooling of sublexical orthographic information that is thought to drive letter migration errors.
\end{abstract}

Keywords Orthographic processing $\cdot$ Letter migrations $\cdot$ Parallel processing $\cdot$ Flanker paradigm $\cdot$ Letter positions

\section{Introduction}

Much of psycholinguistic research has focused on how linguistic processes can operate perfectly, but it may be equally interesting to look at how processing can go awry. One area where this has been particularly informative is that of language production, with well-known examples of speech errors such as spoonerisms and slips of the tongue providing insights with respect to the basic mechanisms involved in producing speech (e.g., Dell \& Reich, 1981). In a similar vein, looking at errors that are based on written language input might provide insight with respect to orthographic processing and, in particular, with respect to one question that has attracted much attention in recent years: the representation and encoding of

Jonathan Grainger

jonathan.grainger@univ-amu.fr

1 Department of Experimental Psychology, Ghent University, Ghent, Belgium

2 Laboratoire de Psychologie Cognitive, CNRS \& Aix-Marseille University, 3 place Victor Hugo, 13331 Marseille, France

3 Institute for Language, Communication and the Brain, Aix-Marseille University, Marseille, France letter position information (see Grainger, 2008). The present study examines one specific type of error that is related to the representation of letter position information: letter migration errors.

Previously, the paradigm of choice to elicit letter migration errors was a divided attention paradigm (Allport, 1977; Davis \& Bowers, 2004; McClelland \& Mozer, 1986; Mozer, 1983; Shallice \& McGill, 1978). In these studies, participants were briefly presented with two different words, followed by postmasking of those words. After that, one of the words was cued for verbal report (or a single letter cued in certain experiments). A consistent finding is that letter migrations occur to form illusory words - that is, words that were not shown to participants. For example, upon presentation of the words SAND and LANE and cued to report the word on the left, participants would incorrectly report LAND instead of SAND, and significantly more so than when the word on the right was a control word such as BANK (Mozer, 1983). One key finding is that letter migrations are more likely to occur between orthographically similar words than between words that have no letters in common, referred to as the surroundsimilarity effect (McClelland \& Mozer, 1986). Another key finding is that the migrating letter does not have to remain at the same position (e.g., a letter on Position 2 in the noncued 
word could migrate to Position 3 in the cued word; Davis \& Bowers, 2004). These two findings point to a system that (1) is able to integrate orthographic information across different words into a single processing channel (McClelland \& Mozer, 1986), and (2) allows for a certain level of uncertainty or flexibility in letter position coding (Davis \& Bowers, 2004).

However, this evidence for the spatial pooling of orthographic information from different words was obtained in conditions that encourage participants to divide their attention across the two words. One could argue that this is very different from more natural reading behavior where attention is thought to be largely focused on one word at a time (e.g., Reichle, Pollatsek, Fisher, \& Rayner, 1998). However, even in sentence reading, it has been found that word-recognition speed is influenced by the extent to which words are orthographically related to upcoming words (e.g., Angele, Tran, \& Rayner, 2013; Dare \& Shillcock, 2013; Snell, Vitu, \& Grainger, 2017), which suggests either that the spatial pooling of orthographic information proceeds preattentively (e.g., Angele et al., 2013), or that attention is inevitably directed to multiple words at once.

In line with the latter findings, evidence for the spatial pooling of orthographic information spanning multiple stimuli without encouraging divided attention has been obtained using the flanking letters lexical decision task (Dare \& Shillcock, 2013). Here, participants have to perform a lexical decision task on centrally presented words flanked by letters located to the left and to the right of targets and separated from the target by a space. In Dare and Shillcock's (2013) seminal study, the flanking letters were bigrams formed from the first two and last two letters of targets in the related condition (e.g., ro rock ck), and different letters in the unrelated condition (e.g., pa rock th). The important finding here is that not only did related flankers facilitate lexical decisions to target words when the bigrams respected their order in the target but they did so to the same extent when bigram order was switched (e.g., ck rock ro). This finding was replicated by Grainger, Mathôt, and Vitu (2014), who further demonstrated that reversing the order of letters in bigrams (e.g., or rock kc) caused a significant reduction in priming. ${ }^{1}$ Grainger et al. (2014) interpreted these findings within the framework for orthographic processing proposed by Grainger and van Heuven (2004). According to this account, orthographic information from both parafoveal flanker and foveal target stimuli are spatially integrated (pooled) into a single processing channel. Flanking letters can then contribute to the process of target-word identification by either providing a boost in activation to the target word's component

\footnotetext{
${ }^{1}$ Flanker effects have also been found using orthographically related words as flankers (Snell, Bertrand, Meeter, \& Grainger, 2018; Snell, Vitu, \& Grainger, 2017). Furthermore, studies comparing the effects of repeated word flankers with both an unrelated flanker and a no-flanker condition (Snell \& Grainger, 2018) have highlighted the contribution of both facilitatory and inhibitory influences of flanking stimuli.
}

letters (in the case of related flankers, thus leading to facilitation) or providing negative evidence for the target word in the case of unrelated flankers, hence leading to inhibition. Here, it is important to note that Grainger et al.'s (2014) account of spatial pooling of orthographic information based on evidence from the flanker paradigm embodies the two main conclusions derived from studies of letter migration errors using the divided attention paradigm: namely, that orthographic information is pooled beyond single words, and that letter position coding is subject to a certain amount of flexibility.

Given that results obtained with the flanker paradigm reflect spatial pooling of sublexical orthographic information (i.e., letters and/or letter combinations), and that the flanker paradigm and the divided attention paradigm seem to provide different windows on the same process, we therefore predicted that we should be able to observe letter migration errors in the flanker paradigm. The current study was designed to test this prediction by using stimuli mimicking those tested in one prior letter migration study (Davis \& Bowers, 2004), and adapting the flanker paradigm to the brief presentation and masking conditions used in prior letter migration experiments. Finding letter migration errors in the flanker paradigm would provide valuable evidence that prior reports of letter migration errors are not due to any specificities of the paradigm that was used, and in particular that such errors did not occur because participants were encouraged to divide their attention across multiple words. The use of target-word identification rather than lexical decision as a task would further yield a more direct view on how the word-recognition process is influenced by surrounding information. Whereas lexical decisions in our previous flanker experiments arguably gave insight with respect to how flankers influenced the experienced "wordlikeness" of target stimuli, a target identification task will tell us concretely whether the spatial pooling of orthographic information can indeed lead to erroneous recognition.

In the present study, participants had to identify a single, centrally located target word that was flanked by the same flanking word to the left and to the right. We manipulated the orthographic overlap across target and flankers in order to induce migration errors. For example, if the following target and flankers, folie farce folie, are presented, the letter $o$ in folie can migrate to replace the letter $a$ in farce thus inducing the migration error force. Following Davis and Bowers (2004), we also investigated migrations to different letter positions in target and flankers.

\section{Method}

\section{Participants}

Fifty-six students (47 female) from Aix-Marseille University gave written consent to partake in this experiment and 
received monetary compensation (at the rate of $€ 10$ /hour) or course credit. All participants were native French speakers, reported to have normal or corrected-to-normal vision, and ranged in age from 18 to 32 years $(M=22.1, S D=3.5)$.

\section{Stimuli and design}

We selected 180 five-letter target-flanker word pairs from the French Lexicon Project database (Ferrand et al., 2010). The targets had a mean frequency of $3.86 \mathrm{ZipF}$ (van Heuven, Mandera, Keuleers, \& Brysbaert, 2014) and were specifically chosen such that if a critical letter was replaced with a different letter drawn from the corresponding flanker word (referred to as the migration flanker), a new word could be formed, referred to as the illusory word (e.g., the replacing the second letter in the target word farce with the second letter from the flanker word folie produces the illusory word force). Illusory words were therefore orthographic neighbors of the target words. Control flanker words were chosen so that no possible combination of letter migrations could result in an existing five-letter French word. All target, migration flanker, and control flanker word triplets had the same initial and final letters, with migrations only possible at Positions 2, 3, or 4 (see Table 1). The target words were from the following grammatical categories: nouns (64\%), verbs (29\%), and adjectives or prepositions (7\%). There were no diacritics (e.g., $\dot{a}, \dot{e}, \ddot{i}, \hat{u}, c ̧$ ) in either the target, flanker, or illusory words. On each trial, the target word was flanked to the left and to the right by either the corresponding migration flanker word or by the matched control word. A small percentage of words could appear twice (e.g., a word that was a target in one trial could be a flanker or an illusory word in another), but no word appeared as a target more than once per condition. We further manipulated the distance (in number of letters) separating the position of the migrating letter in the migration flanker word and in the illusory word. This distance could either be 0 (same position), 1 (adjacent position), or 2 (distant position; see Table 1 for examples). This resulted in a 2 (migration flanker vs. control flanker) $\times 3$ (distance) factorial design. The average target word/migration flanker word/illusory word frequencies $(\mathrm{Zipf})^{2}$ in the three distance conditions were 3.86/4.10/4.12; 4.00/3.78/3.99; 3.72/3.63/3.87. All target words were seen twice by all participants - once with the migration flankers and once with the control flankers. Each set of target words was split in two, and two stimulus lists were created such that in each list half of the targets were paired with a migration flanker and the other half with a

\footnotetext{
${ }^{2}$ It should be noted that $28.05 \%$ of the migration flankers and $11.67 \%$ of the illusory words did not have an entry in the Lexique database and therefore were not included in these calculations. Pair-wise $t$ tests revealed no significant differences between the different migration positions ( $p s>.05)$.
}

control flanker using a Latin-square design. The presentation order of the two lists was counterbalanced across participants. The experiment thus consisted of 360 trials, presented to participants in random order.

\section{Apparatus}

The stimuli and experimental design were implemented with OpenSesame (Mathôt, Schreij, \& Theeuwes, 2012) and presented on a 24 -inch $1,024 \times 768$-pixel LCD-screen. Participants were seated at an 80 -cm distance from the display, so that each character space subtended $0.24^{\circ}$ of visual angle. All words were presented in lowercase using a 24-point monospaced font (droid sans mono, the standard in OpenSesame). All responses were collected via a computer keyboard.

\section{Procedure}

Participants were seated in a comfortable office chair in a dimly lit room. Before the experiment, instructions were given both verbally and visually on-screen. Every trial began with vertically aligned fixation bars that stayed on-screen for 500 $\mathrm{ms}$. After that, the target word and flankers appeared for $50 \mathrm{~ms}$ (each word separated by a single character space). Both target and flanker words were then replaced by masks that consisted of five hash marks ('\#’), which stayed on-screen for $200 \mathrm{~ms}$. After that, a target box appeared one line below the central word in which participants could type their response. Responses could be corrected if necessary using the backspace key. After registering their responses using the return key, a new trial would start (see Fig. 1 for a summary of the procedure). Before the main experiment, 10 practice trials were presented that gave feedback in the form of a green (correct) or red (incorrect) dot. No feedback was given during the main experiment. Participants were offered a break at the halfway point. The experiment lasted approximately 25 minutes.

\section{Results}

The overall average error rate was $23.37 \%(S D=8.46 \%)$. Incorrect responses were categorized either as a letter migration, a word migration, a neighbor migration, or other error. Letter migration errors constitute the report of an illusory word (i.e., a word formed by replacing a letter in the target word with a letter from the migration flanker word, such as reporting SHARE instead of the target SHAME when the flanker is SCARE). ${ }^{3}$ A word migration error occurred when the flanker word was reported instead of the target, and a

\footnotetext{
${ }^{3}$ English examples taken from Davis and Bowers (2004).
} 
Table 1 Examples of stimuli tested in the experiment

\begin{tabular}{|c|c|c|c|c|c|}
\hline Distance & From $\rightarrow$ To position & Target word & Migration flanker & Control flanker & Illusory word \\
\hline \multirow[t]{2}{*}{0 (same) } & $2 \rightarrow 2$ & NUIIRE & NOBBLE & NETTE & NOIRE \\
\hline & $4 \rightarrow 4$ & COULE & CAMPE & CACHE & COUPE \\
\hline \multirow[t]{2}{*}{1 (adjacent) } & $2 \rightarrow 3$ & HALTE & HüILE & HORDE & HAŪTE \\
\hline & $4 \rightarrow 3$ & TIGRE & TANTE & TOQUE & TITRE \\
\hline \multirow[t]{2}{*}{2 (distant) } & $2 \rightarrow 4$ & PLATS & PNEUS & PEURS & PLANS \\
\hline & $4 \rightarrow 2$ & AGILE & ABUSEE & ADORE & ASILE \\
\hline
\end{tabular}

Note. Distance refers to the number of letter positions $(0,1,2)$ separating the position of the migrating letter in the flanker and the illusory word. Position $(2,3,4)$ refers to the position in the flanker (from) and position in the illusory word (to). The migrating letter and the corresponding letter in the target is underlined in these examples for illustration purposes

neighbor migration error occurred when an existing word was reported that is an orthographic neighbor of the flanker word (e.g., reporting SCORE when the target is SHAME and the flanker is SCARE). The remaining errors were classified as "other" and consisted of four-letter words, pseudowords, spelling errors (i.e., five-letter words orthographically similar to the target word), blank responses, or completely different five-letter words. A detailed break-down of these percentages is shown in Table 2.

We used generalized linear mixed models to analyze differences in error rates across conditions, with participants and items as crossed random effects (Baayen, Davidson, \& Bates, 2008; Barr, Levy, Scheepers, \& Tily, 2013). This was done using the glmer function from the lme4 package (Bates, Mächler, Bolker, \& Walker, 2015). We report regression coefficients $(b)$, standard errors $(S E)$ and $z$ values. Fixed effects were deemed reliable if $|z|>1.96$ (Baayen, 2008). All analyses were done with the Rstudio (Version 3.4.2) statistical computing environment. We focus on letter migration errors and only report other effects when significant.

\section{Letter migration errors}

We observed a total of 361 word reports out of all the trials from the letter migration condition (which equals $3.57 \%$ ). At first sight, this number might look rather small, but bear in mind that the majority of trials were answered correctly $(75.87 \%)$. To examine whether our manipulation was successful at inducing letter migrations we looked at the number of times an illusory word was reported as a result of our manipulation (i.e., with a migration flanker) versus when it was reported as an error that could not have resulted from a migration (the control condition). We also examined the impact of the distance between the position the migratory letter occupied in the flanker versus the position it occupied in the illusory word (same, adjacent, distant: see Table 1 for examples, and Table 3 for results). We found a significant main effect of condition $(b=-0.86, S E=0.10, z=-8.68)$, meaning that an illusory word was more likely to be reported in the migration condition. ${ }^{4}$ We also observed a main effect of distance $(b=$ $-1.99, S E=0.39, z=-5.12)$. Crucially, there was a significant interaction between condition and distance $(b=-1.95, S E=$ $0.25, z=-7.63){ }^{5}$ As can be seen in Table 3, the interaction reflects the monotonic decrease in the size of letter migration effects (migration - control) as a function of distance. It also reflects the fact that the effect of distance was significant in the migration condition $(b=-2.38, S E=0.49, z=-4.87)$, but not in the control condition $(b=-0.62, S E=0.48, z=-1.29)$.

\section{Word migration errors}

Comparing the number of flanker words reported instead of target words in the letter migration and control conditions revealed a significant effect of condition $(b=0.18, S E=$ $0.08, z=2.11$ ), with more flanker words being reported in the control condition.

\section{Frequency effects}

In a final analysis, we examined the effects of word frequency on the number of illusory word reports due to letter migrations. The frequency values (Zipf) for the target words, the illusory words, the migration flanker words, and the control flanker words were entered as continuous variables along with the condition variable. The results for the target and illusory words are shown in Table 5. Flanker word frequency had no significant influence (migration flanker: $b=-0.15, S E=0.23$, $z=-0.65$; control flanker: $b=-0.29, S E=0.20, z=-1.47$ ). As can be seen in Table 4 , there was no main effect of target-word

\footnotetext{
${ }^{4}$ Presentation order (i.e., having seen the target word in the letter migration or control condition first) did not have a significant influence $(b=-0.07, S E=$ $0.09, z=-0.81)$ and did not interact with the effect of condition $(b=0.45, S E=$ $0.25, z=1.77)$. Furthermore, because some illusory words did appear as targets $(N=23)$, we examined whether seeing the word in advance made a difference in misreporting it as an illusory word. No significant effect was observed $(b=$ $22.33, S E=26.69, z=0.83$ ). It should also be noted that the average distance between an illusory word seen before as a target and that illusory word being reported was 87 trials.

5 The same pattern of effects was obtained when including word frequency as a continuous variable in the LME analyses.
} 


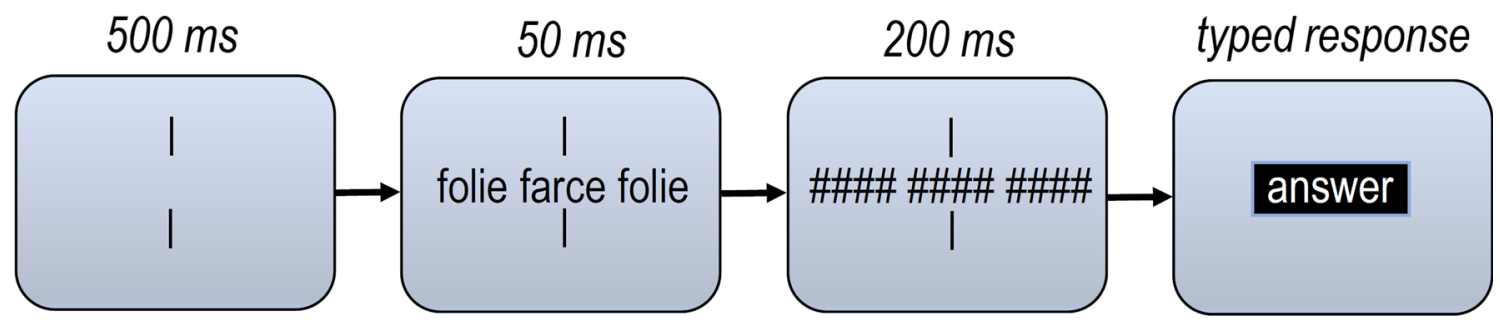

Fig. 1 Procedure of the flanker task used in the present study. Participants had to type in the identity of the central target word

frequency, but a significant interaction with condition. Targetword frequency only affected illusory word reports in the control condition. There was a main effect of illusory word frequency and an interaction with condition. Illusory word frequency had a significant impact on illusory word reports in both conditions, but the effect was greater in the control condition.

\section{Discussion}

The main goal of the present experiment was to examine whether letter migration errors can be observed in a flanker paradigm in which target words are centrally located and where focusing all attentional resources solely on the target would be beneficial for the task. The aim was to demonstrate that prior observations of letter migration errors using a divided attention paradigm (e.g., Davis \& Bowers, 2004; McClelland \& Mozer, 1986; Mozer, 1983) were not the result of processes triggered by the fact that participants were encouraged to identify two words at the same time in these studies. Our results suggest, indeed, that this was not the case, because we were successful in inducing letter migration errors in the flanker paradigm where only one word has to be identified. Here, we will argue that it is the spatial pooling of sublexical orthographic information that is the cause of letter migration errors seen in both the divided attention paradigm and the flanker paradigm.

An important finding of the present experiment is that we observed a level of letter migration errors that

Table 2 Average percentages of response types per condition

\begin{tabular}{lll}
\hline Condition & & \\
\hline Response type & Migration & Control \\
\hline Correct & 75.87 & 77.29 \\
Letter migration & 3.57 & 1.69 \\
Word migration & 2.78 & 3.23 \\
Neighbor migration & 0.08 & 0.17 \\
Other & 17.68 & 17.62 \\
\hline
\end{tabular}

is comparable with that found in the third experiment of Davis and Bowers (2004), upon which the present experiment was based. Although Davis and Bowers (2004) observed a greater percentage of letter migration errors overall, the relative error rate (relative to the total amount of errors) was comparable in their Experiment 3 and our experiment (18.83\% vs. $14.85 \%)$. This simply suggests that it is harder to identify target words in the divided attention paradigm than in the flanker paradigm, leading to more errors overall, but that a similar mechanism is driving letter migration errors in the two paradigms, thus leading to a similar percentage of this type of error.

The second important finding of the present study concerns differences in the size of the letter migration effect as a function of the distance (in number of letters) between the position of the migrating letter in the flanker and the illusory words $(0,1$, or 2$)$. We found that the letter migration effect significantly diminished as the distance increased, to the point that it disappeared with the distant (two letter position difference) migrations (see Table 3). Davis and Bowers (2004) reported a similar monotonic decrease in the letter migration effect as a function of distance, but nevertheless found a significant effect of letter migrations with distant migrations. The fact that we failed to find an effect with distant migrations is likely due to the overall lower number of letter migrations obtained in the flanker paradigm. The key finding, nevertheless, is the significant interaction between the letter migration effect and migration distance. This finding fits with most current models of letter position coding (Davis, 2010; Dehaene, Cohen, Sigman, \& Vinckier, 2005; Gomez, Ratcliff, \& Perea, 2008; Grainger \& van Heuven, 2004; Whitney, 2001), except for an unconstrained open-bigram model. Within the framework of open-bigram coding, used by Grainger et al. (2014) to account for spatial pooling of orthographic information, this pattern of results suggests either that more weight should be assigned to contiguous bigrams than to noncontiguous ones, or that an unconstrained open-bigram code must be complemented with a more precise position-coding mechanism using word edges (see Grainger, Dufau, \& Ziegler, 2016; Snell, Bertrand, \& Grainger, 2018). 
Table 3 Letter migration effects as a function of migration distance

\begin{tabular}{lccccc}
\hline Migration distance & Migration & Control & Difference & $b$ & \multicolumn{2}{c}{$S E$} & $-\mathbf{1 0 . 4 2}$ \\
\hline 0 (same) & 6.90 & 1.64 & 5.26 & -1.67 & 0.16 \\
1 (adjacent) & 2.14 & 1.39 & 0.75 & -0.44 & 0.19 \\
2 (distant) & 1.64 & 1.99 & -0.35 & 0.23 & 0.19 \\
\hline
\end{tabular}

Note. Data are the average percentage illusory word reports with letter migration flankers (Migration) and control flankers (Control). Migration distance $(0,1,2)$ represents the number of letter positions separating the position of the migrating letter in the flanker and its position in the illusory word. Significant values are shown in bold

A third important finding is that frequency plays a crucial role in the reporting of illusory words (see Table 4). Given that the illusory word is an orthographic neighbor of the target, the influence of illusory word frequency can be taken as evidence that the higher the frequency of an orthographic neighbor, the more strongly it will be activated upon presentation of the target word and hence be incorrectly reported instead of the target word. This is simply another demonstration of the impact of highfrequency orthographic neighbors on target-word processing in data-limited identification tasks (Carreiras, Perea, \& Grainger, 1997; Grainger \& Jacobs, 1996; Grainger \& Segui, 1990). It is important to note, however, that there is abundant evidence that the interfering effects of orthographic neighbors are also obtained in speed of responding in response-limited paradigms (e.g., Carreiras et al., 1997; Grainger, 1990) as well as with eyemovement recordings in a simplified reading task (Grainger, O'Regan, Jacobs, \& Segui, 1989) and during sentence reading (e.g., Perea \& Pollatsek, 1998). This suggests that orthographic neighbors are influencing online processing of target words and not just processes that are implemented when word identification fails. The general idea is that the lexical representations of orthographic neighbors can be activated in parallel with the target-word representation and compete for identification (see Grainger \& Jacobs, 1996, for further discussion). Although significant effects of illusory word frequency were found in both the migration and control flanker conditions, the effect was significantly stronger in the control condition. This suggests that the contribution of letter migrations to illusory word reports somewhat dampened the impact of illusory word frequency on such reports, and fits well with our interpretation of letter migration errors as reflecting online orthographic processing of target words.

Finally, we also found more word migrations in the control condition than in the letter migration condition. This is most likely due to the greater evidence for the illusory word, compared with the flanker word, in the letter migration condition. This is the very basis of the letter migration effect. In other words, illusory words are reported more than flanker words when these illusory words can be formed (i.e., in the letter migration condition), hence reducing the overall report of flanker words in that condition. Similarly, we found that target-word frequency only had an impact on accuracy in the control condition. This again could be due to the influence of illusory word frequency reducing the impact of target word frequency in the letter migration condition.

Is there evidence for spatial pooling of orthographic information in more natural reading situations? The answer is clearly yes. As noted in the Introduction, Dare and Shillcock (2013) not only demonstrated such effects in the flanker paradigm but also in a sentence-reading experiment with eyemovement recordings. This was done by manipulating the orthographic overlap between the currently fixated word (the target) and the letter string immediately to its right (the parafoveal stimulus). Once readers' eyes left the critical target word, the parafoveal stimulus became the normal continuation of the sentence. Thus, for example, participants read the following word sequence: "The store had a coat coat that week," and when their eyes left the first occurrence of "coat," the second occurrence was changed to "sale," and participants had the impression they had read the syntactically correct sentence "The store had a coat sale that week." This repetition condition was compared with "The store had a coat milk that week," with the word "milk" changing to "sale" as readers' eyes left the word "coat." Dare and Shillcock found that target word viewing times were significantly reduced when the

Table 4 Effects of target word frequency and illusory word frequency on illusory word reports

\begin{tabular}{|c|c|c|c|c|c|c|}
\hline & \multicolumn{3}{|c|}{ Target word } & \multicolumn{3}{|c|}{ Illusory word } \\
\hline & $b$ & $S E$ & $z$ & $b$ & $S E$ & $z$ \\
\hline Frequency $(\mathrm{F})$ & -0.24 & 0.17 & -1.43 & 1.19 & 0.19 & 6.19 \\
\hline Condition $\mathrm{x} F$ & -0.48 & 0.11 & -4.39 & -0.37 & 0.16 & -2.28 \\
\hline F/Migration & -0.12 & 0.26 & -0.51 & 0.79 & 0.24 & 3.29 \\
\hline F/Control & -0.90 & 0.20 & -4.45 & 0.86 & 0.23 & 3.75 \\
\hline
\end{tabular}

Note. The two bottom lines show the effects of target word and illusory word frequency separately for the migration and control conditions. Effects of flanker word frequency were not significant $(z<1.5)$. Significant values are shown in bold 
parafoveal stimulus was a repetition of the target, and also when it was an orthographically similar pseudoword (e.g., "coat"-"caot"; see Angele et al., 2013; Snell et al., 2017, for further evidence obtained in sentence reading with eye movements and with words and pseudowords that are orthographic neighbors). Given this evidence, we suspect that letter migrations are part and parcel of the normal process of reading.

To conclude, the present finding that letter migration errors occur in the flanker paradigm lends support to two major conclusions drawn on the basis of findings obtained with the divided attention paradigm: (1) that sublexical orthographic information is processed in parallel across distinct stimuli and spatially integrated into a single processing channel (McClelland \& Mozer, 1986), and (2) that this pooling process operates on an orthographic code in which letter identities are not strictly associated with specific positions in a word (Davis \& Bowers, 2004).

Acknowledgements The present research was performed while A.V. was on an Erasmus exchange between Ghent University and Aix-Marseille University. This research was supported by ERC Grant 742141 . We thank Charlotte Leflaëc for her help in running the experiment.

\section{Appendix}

Table 5 List of stimuli

\begin{tabular}{|c|c|c|c|c|}
\hline Target word & Position (from $\rightarrow$ to) & Migration flanker & Control flanker & Illusory word \\
\hline Fondu & $2 \rightarrow 2$ & Fessu & Fallu & Fendu \\
\hline Ruses & $2 \rightarrow 2$ & Ronds & Rails & Roses \\
\hline Cache & $2 \rightarrow 2$ & Coure & Curie & Coche \\
\hline Sobre & $2 \rightarrow 2$ & Salue & Selle & Sabre \\
\hline Nuire & $2 \rightarrow 2$ & Noble & Nette & Noire \\
\hline Malle & $2 \rightarrow 2$ & Mitre & Malte & Mille \\
\hline Fosse & $2 \rightarrow 2$ & Femme & Farde & Fesse \\
\hline Plume & $2 \rightarrow 2$ & Parie & Pense & Paume \\
\hline Pinte & $2 \rightarrow 2$ & Poule & Palpe & Ponte \\
\hline Farce & $2 \rightarrow 2$ & Folie & Feule & Force \\
\hline Bases & $2 \rightarrow 2$ & Biens & Bonus & Bises \\
\hline Clope & $2 \rightarrow 2$ & Chute & Carte & Chope \\
\hline Roche & $2 \rightarrow 2$ & Rifle & Rende & Riche \\
\hline Songe & $2 \rightarrow 2$ & Situe & Selle & Singe \\
\hline Vaste & $2 \rightarrow 2$ & Verre & Viole & Veste \\
\hline Batte & $2 \rightarrow 2$ & Boire & Beure & Botte \\
\hline Mises & $2 \rightarrow 2$ & Mucus & Morts & Muses \\
\hline Foire & $2 \rightarrow 2$ & Faune & Fende & Faire \\
\hline Laque & $2 \rightarrow 2$ & Lotie & Lippe & Loque \\
\hline Pages & $2 \rightarrow 2$ & Pious & Ponts & Piges \\
\hline Rires & $2 \rightarrow 2$ & Rangs & Ronds & Rares \\
\hline Rites & $2 \rightarrow 2$ & Rangs & Ruons & Rates \\
\hline Peste & $2 \rightarrow 2$ & Pille & Parle & Piste \\
\hline Soins & $2 \rightarrow 2$ & Sales & Sures & Sains \\
\hline Parle & $2 \rightarrow 2$ & Poste & Pelte & Poire \\
\hline Vague & $2 \rightarrow 2$ & Voire & Veste & Vogue \\
\hline Jaune & $2 \rightarrow 2$ & Jette & Jolie & Jeune \\
\hline Brins & $2 \rightarrow 2$ & Bauds & Buses & Bains \\
\hline Sport & $2 \rightarrow 2$ & Shift & Salut & Short \\
\hline Cible & $2 \rightarrow 2$ & Carpe & Coche & Cable \\
\hline Renie & $4 \rightarrow 4$ & Ruade & Racle & Rende \\
\hline Taupe & $4 \rightarrow 4$ & Tuile & Trime & Taule \\
\hline Sonde & $4 \rightarrow 4$ & Sarge & Sable & Songe \\
\hline Guise & $4 \rightarrow 4$ & Garde & Gomme & Guide \\
\hline Frite & $4 \rightarrow 4$ & Femme & Fauve & Frime \\
\hline Parts & $4 \rightarrow 4$ & Plocs & Pieds & Parcs \\
\hline Filme & $4 \rightarrow 4$ & Foule & Farde & Fille \\
\hline Phare & $4 \rightarrow 4$ & Pulse & Pompe & Phase \\
\hline Moine & $4 \rightarrow 4$ & Meute & Marge & Moite \\
\hline Bribe & $4 \rightarrow 4$ & Blase & Balle & Brise \\
\hline Marge & $4 \rightarrow 4$ & Momie & Menue & Marie \\
\hline Stade & $4 \rightarrow 4$ & Songe & Serpe & Stage \\
\hline Meure & $4 \rightarrow 4$ & Mixte & Magie & Meute \\
\hline
\end{tabular}


Table 5 (continued)

\begin{tabular}{|c|c|c|c|c|}
\hline Target word & Position (from $\rightarrow$ to) & Migration flanker & Control flanker & Illusory word \\
\hline Trous & $4 \rightarrow 4$ & Tapis & Temps & Trois \\
\hline Serve & $4 \rightarrow 4$ & Sobre & Saine & Serre \\
\hline Coule & $4 \rightarrow 4$ & Campe & Cache & Coupe \\
\hline Fonds & $4 \rightarrow 4$ & Faits & Fumes & Fonts \\
\hline Halle & $4 \rightarrow 4$ & Honte & Heure & Halte \\
\hline Pairs & $4 \rightarrow 4$ & Puons & Pouls & Pains \\
\hline Exige & $4 \rightarrow 4$ & Enfle & Entre & Exile \\
\hline Garce & $4 \rightarrow 4$ & Guide & Golfe & Garde \\
\hline Douze & $4 \rightarrow 4$ & Dance & Demie & Douce \\
\hline Larve & $4 \rightarrow 4$ & Longe & Loupe & Large \\
\hline Barbe & $4 \rightarrow 4$ & Boire & Biffe & Barre \\
\hline Bagne & $4 \rightarrow 4$ & Bique & Bille & Bague \\
\hline Taule & $4 \rightarrow 4$ & Tempe & Terme & Taupe \\
\hline Plans & $4 \rightarrow 4$ & Ports & Poses & Plats \\
\hline Soupe & $4 \rightarrow 4$ & Sente & Sache & Soute \\
\hline Poids & $4 \rightarrow 4$ & Pulls & Parts & Poils \\
\hline Crise & $4 \rightarrow 4$ & Comme & Cafte & Crime \\
\hline Prise & $2 \rightarrow 3$ & Poche & Peche & Prose \\
\hline Merle & $2 \rightarrow 3$ & Munie & Mante & Meule \\
\hline Coups & $2 \rightarrow 3$ & Cries & Cabas & Corps \\
\hline Aille & $2 \rightarrow 3$ & Agace & Abuse & Aigle \\
\hline Folle & $2 \rightarrow 3$ & Furie & Faire & Foule \\
\hline Crame & $2 \rightarrow 3$ & Cible & Chose & Crime \\
\hline Saule & $2 \rightarrow 3$ & Slice & Sobre & Salle \\
\hline Sorte & $2 \rightarrow 3$ & Suive & Salve & Soute \\
\hline Onlce & $2 \rightarrow 3$ & Ogive & Offre & Ongle \\
\hline Grave & $2 \rightarrow 3$ & Gilde & Gosse & Grive \\
\hline Ouvre & $2 \rightarrow 3$ & Otage & Opine & Outre \\
\hline Payer & $2 \rightarrow 3$ & Prier & Polir & Parer \\
\hline Faite & $2 \rightarrow 3$ & Fusse & Ferme & Faute \\
\hline Halte & $2 \rightarrow 3$ & Huile & Horde & Haute \\
\hline Tapie & $2 \rightarrow 3$ & Troue & Tombe & Tarie \\
\hline Corps & $2 \rightarrow 3$ & Cubes & Chics & Coups \\
\hline Ville & $2 \rightarrow 3$ & Votre & Vache & Viole \\
\hline Toits & $2 \rightarrow 3$ & Trams & Temps & Torts \\
\hline Fixer & $2 \rightarrow 3$ & Fluor & Futur & Filer \\
\hline Pacte & $2 \rightarrow 3$ & Probe & Poile & Parte \\
\hline Hurle & $2 \rightarrow 3$ & Hisse & Hache & Huile \\
\hline Dinde & $2 \rightarrow 3$ & Dogue & Dague & Diode \\
\hline Outre & $2 \rightarrow 3$ & Ovale & Oigne & Ouvre \\
\hline Sages & $2 \rightarrow 3$ & Slows & Sinus & Sales \\
\hline Fumes & $2 \rightarrow 3$ & Finis & Fards & Fuies \\
\hline Coton & $2 \rightarrow 3$ & Clown & Caban & Colon \\
\hline Plaie & $2 \rightarrow 3$ & Purge & Ponce & Pluie \\
\hline Suave & $2 \rightarrow 3$ & Singe & Sobre & Suive \\
\hline Marre & $2 \rightarrow 3$ & Mufle & Morse & Maure \\
\hline Filme & $2 \rightarrow 3$ & Frite & Fasse & Firme \\
\hline Capte & $4 \rightarrow 3$ & Cuire & Chime & Carte \\
\hline Tigre & $4 \rightarrow 3$ & Tante & Toque & Titre \\
\hline Borde & $4 \rightarrow 3$ & Bague & Basse & Boude \\
\hline Pompe & $4 \rightarrow 3$ & Parue & Pense & Poupe \\
\hline Pouce & $4 \rightarrow 3$ & Peine & Paire & Ponce \\
\hline Liens & $4 \rightarrow 3$ & Labos & Lupus & Lions \\
\hline Piges & $4 \rightarrow 3$ & Pouls & Ponds & Piles \\
\hline Dette & $4 \rightarrow 3$ & Drone & Dance & Dente \\
\hline Vides & $4 \rightarrow 3$ & Volts & Vapes & Vites \\
\hline Verte & $4 \rightarrow 3$ & Visse & Voice & Veste \\
\hline Chats & $4 \rightarrow 3$ & Cocus & Coins & Chuts \\
\hline Texte & $4 \rightarrow 3$ & Turne & Table & Tente \\
\hline Mette & $4 \rightarrow 3$ & Magne & Malle & Mente \\
\hline Votes & $4 \rightarrow 3$ & Vrais & Vapes & Voies \\
\hline Rames & $4 \rightarrow 3$ & Rhums & Ronds & Rares \\
\hline Parme & $4 \rightarrow 3$ & Pique & Ponde & Paume \\
\hline Beige & $4 \rightarrow 3$ & Boule & Bande & Belge \\
\hline Verge & $4 \rightarrow 3$ & Vaine & Vomie & Venge \\
\hline
\end{tabular}


Table 5 (continued)

\begin{tabular}{|c|c|c|c|c|}
\hline Target word & Position (from $\rightarrow$ to) & Migration flanker & Control flanker & Illusory word \\
\hline Verve & $4 \rightarrow 3$ & Vaque & Vanne & Veuve \\
\hline Amant & $4 \rightarrow 3$ & Argot & Assit & Amont \\
\hline Sable & $4 \rightarrow 3$ & Situe & Serpe & Saule \\
\hline Salve & $4 \rightarrow 3$ & Situe & Sente & Sauve \\
\hline Moque & $4 \rightarrow 3$ & Mitre & Mince & Morue \\
\hline Pause & $4 \rightarrow 3$ & Peine & Poche & Panse \\
\hline Lieue & $4 \rightarrow 3$ & Lange & Lampe & Ligue \\
\hline Dames & $4 \rightarrow 3$ & Doits & Dicos & Dates \\
\hline Chars & $4 \rightarrow 3$ & Codes & Colis & Chers \\
\hline Reste & $4 \rightarrow 3$ & Ruine & Rafle & Rente \\
\hline Avant & $4 \rightarrow 3$ & Admet & Argot & Avent \\
\hline Potes & $4 \rightarrow 3$ & Pairs & Panas & Pores \\
\hline Crocs & $2 \rightarrow 4$ & Ciels & Cales & Crois \\
\hline Tract & $2 \rightarrow 4$ & Tient & Twist & Trait \\
\hline Celte & $2 \rightarrow 4$ & Clame & Cadre & Celle \\
\hline Avale & $2 \rightarrow 4$ & Arche & Aboie & Avare \\
\hline Loups & $2 \rightarrow 4$ & Lents & Laids & Loues \\
\hline Bulbe & $2 \rightarrow 4$ & Blase & Barde & Bulle \\
\hline Parai & $2 \rightarrow 4$ & Ponti & Publi & Paroi \\
\hline Proie & $2 \rightarrow 4$ & Pulpe & Palme & Proue \\
\hline Tract & $2 \rightarrow 4$ & Tient & Tuent & Trait \\
\hline Rende & $2 \rightarrow 4$ & Riche & Rampe & Renie \\
\hline Venge & $2 \rightarrow 4$ & Vulve & Vampe & Venue \\
\hline Maths & $2 \rightarrow 4$ & Meurs & Munis & Mates \\
\hline Plats & $2 \rightarrow 4$ & Pneus & Peuts & Plans \\
\hline Porcs & $2 \rightarrow 4$ & Peins & Palis & Pores \\
\hline Chaos & $2 \rightarrow 4$ & Crues & Cries & Chars \\
\hline Mines & $2 \rightarrow 4$ & Muscs & Motos & Minus \\
\hline Tarte & $2 \rightarrow 4$ & Tique & Toute & Tarie \\
\hline Remet & $2 \rightarrow 4$ & Riant & Ragot & Remit \\
\hline Orale & $2 \rightarrow 4$ & Ogive & Ozone & Orage \\
\hline Chefs & $2 \rightarrow 4$ & Crans & Coups & Chers \\
\hline Salue & $2 \rightarrow 4$ & Singe & Score & Salie \\
\hline Boude & $2 \rightarrow 4$ & Blase & Berne & Boule \\
\hline Soirs & $2 \rightarrow 4$ & Seuls & Sauts & Soies \\
\hline Plais & $2 \rightarrow 4$ & Pneus & Pures & Plans \\
\hline Couve & $2 \rightarrow 4$ & Clame & Campe & Coule \\
\hline Diras & $2 \rightarrow 4$ & Dents & Duels & Dires \\
\hline Parte & $2 \rightarrow 4$ & Pulse & Pende & Parue \\
\hline Finis & $2 \rightarrow 4$ & Feras & Fards & Fines \\
\hline Salis & $2 \rightarrow 4$ & Serfs & Shows & Sales \\
\hline Tente & $2 \rightarrow 4$ & Tuile & Tarte & Tenue \\
\hline Volet & $4 \rightarrow 2$ & Vivat & Vingt & Valet \\
\hline Arbre & $4 \rightarrow 2$ & Azyme & Acide & Ambre \\
\hline Types & $4 \rightarrow 2$ & Tubas & Tests & Tapes \\
\hline Agent & $4 \rightarrow 2$ & Assit & Ajout & Aient \\
\hline Fasse & $4 \rightarrow 2$ & Figue & Fibre & Fusse \\
\hline Armes & $4 \rightarrow 2$ & Abois & Avons & Aimes \\
\hline Mains & $4 \rightarrow 2$ & Matos & Muses & Moins \\
\hline Peint & $4 \rightarrow 2$ & Pavot & Parut & Point \\
\hline Puise & $4 \rightarrow 2$ & Phare & Pagne & Prise \\
\hline Menus & $4 \rightarrow 2$ & Mugis & Maths & Minus \\
\hline Pries & $4 \rightarrow 2$ & Pumas & Ponts & Paies \\
\hline Divin & $4 \rightarrow 2$ & Doyen & Dugon & Devin \\
\hline Ruant & $4 \rightarrow 2$ & Remit & Robot & Riant \\
\hline Tendu & $4 \rightarrow 2$ & Tabou & Tribu & Tondu \\
\hline Cuves & $4 \rightarrow 2$ & Comas & Coups & Caves \\
\hline Tares & $4 \rightarrow 2$ & Tunis & Thons & Tires \\
\hline Ravis & $4 \rightarrow 2$ & Roues & Ruons & Revis \\
\hline Pendu & $4 \rightarrow 2$ & Pilou & Panse & Pondu \\
\hline Tuent & $4 \rightarrow 2$ & Trait & Tarot & Tient \\
\hline Mener & $4 \rightarrow 2$ & Mugir & Major & Miner \\
\hline Agile & $4 \rightarrow 2$ & Abuse & Adore & Asile \\
\hline Moche & $4 \rightarrow 2$ & Marie & Masse & Miche \\
\hline Seins & $4 \rightarrow 2$ & Sumos & Surfs & Soins \\
\hline
\end{tabular}


Table 5 (continued)

\begin{tabular}{lllll}
\hline Target word & Position $($ from $\rightarrow$ to) & Migration flanker & Control flanker & Illusory word \\
\hline Aigle & $4 \rightarrow 2$ & Anone & Ardue & Angle \\
Fluet & $4 \rightarrow 2$ & Fagot & Finit & Fouet \\
Pales & $4 \rightarrow 2$ & Punis & Poufs & Piles \\
Pains & $4 \rightarrow 2$ & Puces & Ponds & Peins \\
Fugue & $4 \rightarrow 2$ & Folie & Fable & Figue \\
Antre & $4 \rightarrow 2$ & Avoue & Aboie & Autre \\
Lames & $4 \rightarrow 2$ & Logis & Loups & Limes \\
\hline
\end{tabular}

Open Access This article is distributed under the terms of the Creative Commons Attribution 4.0 International License (http:// creativecommons.org/licenses/by/4.0/), which permits unrestricted use, distribution, and reproduction in any medium, provided you give appropriate credit to the original author(s) and the source, provide a link to the Creative Commons license, and indicate if changes were made.

\section{References}

Allport, D.A. (1977). On the knowing the meaning of words we are unable to report: The effects of visual masking. In S. Dornic (Ed.), Attention and Performance VI (pp. 505-533. Hillsdale, NJ: Erlbaum.

Angele, B., Tran, R., \& Rayner, K. (2013). Parafoveal-foveal overlap can facilitate ongoing word identification during reading: Evidence from eye movements. Journal of Experimental Psychology: Human Perception and Performance, 39(2), 526.

Baayen, R. (2008). Analyzing linguistic data: A practical introduction to statistics. Cambridge, UK: Cambridge University Press.

Baayen, R. H., Davidson, D. J., \& Bates, D. M. (2008). Mixed-effects modeling with crossed random effects for subjects and items. Journal of Memory and Language, 59(4), 390-412. doi:https://doi. org/10.1016/j.jml.2007.12.005

Barr, D. J., Levy, R., Scheepers, C., \& Tily, H. J. (2013). Random effects structure for confirmatory hypothesis testing: Keep it maximal. Journal of Memory and Language, 68(3), 255-278. doi:https://doi. org/10.1016/j.jml.2012.11.001

Bates, D., Mächler, M., Bolker, B., \& Walker, S. (2015). Fitting linear mixed-effects models using lme4. Journal of Statistical Software, 67(1). doi:https://doi.org/10.18637/jss.v067.i01

Carreiras, M., Perea, M., \& Grainger, J. (1997). Effects of orthographic neighborhood in visual word recognition: Cross-task comparisons. Journal of Experimental Psychology: Learning, Memory, and Cognition, 23, 857-871.

Dare, N., \& Shillcock, R. (2013). Serial and parallel processing in reading: Investigating the effects of parafoveal orthographic information on nonisolated word recognition. Quarterly Journal of Experimental Psychology, 66(3), 487-504. doi:https://doi.org/10. 1080/17470218.2012.703212

Davis, C. J. (2010). The spatial coding model of visual word identification. Psychological Review, 117(3), 713-758. doi:https://doi.org/10. 1037/a0019738

Davis, C. J., \& Bowers, J. S. (2004). What do letter migration errors reveal about letter position coding in visual word recognition? Journal of Experimental Psychology: Human Perception and Performance, 30(5), 923-941. doi:https://doi.org/10.1037/00961523.30.5.923
Dehaene, S., Cohen, L., Sigman, M., \& Vinckier, F. (2005). The neural code for written words: A proposal. Trends in Cognitive Sciences, 9(7), 335-341. doi:https://doi.org/10.1016/j.tics.2005.05.004

Dell, G. S., \& Reich, P. A. (1981). Stages in sentence production: An analysis of speech error data. Journal of Verbal Learning and Verbal Behavior, 20(6), 611-629. doi:https://doi.org/10.1016/S00225371(81)90202-4

Ferrand, L., New, B., Brysbaert, M., Keuleers, E., Bonin, P., Méot, A., . . . Pallier, C. (2010). The French Lexicon Project: Lexical decision data for 38,840 French words and 38,840 pseudowords. Behavior Research Methods, 42(2), 488-496. doi:https://doi.org/10.3758/ BRM.42.2.488

Gomez, P., Ratcliff, R., \& Perea, M. (2008). The overlap model: A model of letter position coding. Psychological Review, 115(3), 577-600. doi:https://doi.org/10.1037/a0012667

Grainger, J. (1990). Word frequency and neighborhood frequency effects in lexical decision and naming. Journal of Memory and Language, 29(2), 228-244. doi:https://doi.org/10.1016/0749-596X(90)90074A

Grainger, J. (2008). Cracking the orthographic code: An introduction. Language and Cognitive Processes, 23(1), 1-35. doi:https://doi. org/10.1080/01690960701578013

Grainger, J., Dufau, S., \& Ziegler, J. C. (2016). A vision of reading. Trends in Cognitive Sciences, 20(3), 171-179. doi:https://doi.org/ 10.1016/j.tics.2015.12.008

Grainger, J., \& Jacobs, A. M. (1996). Orthographic processing in visual word recognition: A multiple read-out model. Psychological Review, 103(3), 518-565. doi:https://doi.org/10.1037/0033-295X. 103.3.518

Grainger, J., Mathôt, S., \& Vitu, F. (2014). Tests of a model of multi-word reading: Effects of parafoveal flanking letters on foveal word recognition. Acta Psychologica, 146, 35-40. doi:https://doi.org/10.1016/j. actpsy.2013.11.014

Grainger, J., O’Regan, J. K., Jacobs, A. M., \& Segui, J. (1989). On the role of competing word units in visual word recognition: The neighborhood frequency effect. Perception \& Psychophysics, 45(3), 189-195. doi:https://doi.org/10.3758/ BF03210696

Grainger, J., \& Segui, J. (1990). Neighborhood frequency effects in visual word recognition: A comparison of lexical decision and masked identification latencies. Perception \& Psychophysics, 47(2), 191198. doi:https://doi.org/10.3758/BF03205983

Grainger, J., \& van Heuven, W. J. B. (2004). Modeling letter position coding in printed word perception. In P. Bonin (Ed.), The mental lexicon. New York, NY: Nova Science Publishers.

Mathôt, S., Schreij, D., \& Theeuwes, J. (2012). OpenSesame: An opensource, graphical experiment builder for the social sciences. Behavior Research Methods, 44(2), 314-324. doi:https://doi.org/ 10.3758/s13428-011-0168-7

McClelland, J. L., \& Mozer, M. C. (1986). Perceptual interactions in twoword displays: Familiarity and similarity effects. Journal of 
Experimental Psychology: Human Perception and Performance, 12(1), 18-35. doi:https://doi.org/10.1037/0096-1523.12.1.18

Mozer, M. C. (1983). Letter migration in word perception. Journal of Experimental Psychology: Human Perception and Performance, 9(4), 531-546. doi:https://doi.org/10.1037/0096-1523.9.4.531

Perea, M., \& Pollatsek, A. (1998). The effects of neighborhood frequency in reading and lexical decision. Journal of Experimental Psychology: Human Perception and Performance, 24, 767-779.

Reichle, E. D., Pollatsek, A., Fisher, D. L., \& Rayner, K. (1998). Toward a model of eye movement control in reading. Psychological Review, 105(1), 125-157. doi:https://doi.org/10.1037/0033-295X.105.1.125

Shallice, T. \& McGill, J. (1978). The origins of mixed errors. In J. Requin (Ed.), Attention and Performance VII (pp. 193-208). Hillsdale, NJ: Erlbaum.

Snell, J. \& Grainger, J. (2018). Parallel word processing in the flanker paradigm has a rightward bias. Attention, Perception, \& Psychophysics, 80, 1512-1519.

Snell, J., Bertrand, D., Meeter, M., \& Grainger, J. (2018). Integrating orthographic information across time and space: Masked priming and flanker effects with orthographic neighbors. Experimental
Psychology, 65(1), 32-39. doi:https://doi.org/10.1027/1618-3169/ a000386

Snell, J., Vitu, F., \& Grainger, J. (2017). Integration of parafoveal orthographic information during foveal word reading: Beyond the sublexical level? Quarterly Journal of Experimental Psychology, 70(10), 1984-1996. doi:https://doi.org/10.1080/17470218.2016. 1217247

van Heuven, W. J. B., Mandera, P., Keuleers, E., \& Brysbaert, M. (2014). Subtlex-UK: A new and improved word frequency database for British English. Quarterly Journal of Experimental Psychology, 67(6), 11761190. doi:https://doi.org/10.1080/17470218.2013.850521

Whitney, C. (2001). How the brain encodes the order of letters in a printed word: The SERIOL model and selective literature review. Psychonomic Bulletin \& Review, 8(2), 221-243. doi:https://doi. org/10.3758/BF03196158

Publisher's note Springer Nature remains neutral with regard to jurisdictional claims in published maps and institutional affiliations. 